\title{
An efficient numerical approach to solve the space fractional FitzHugh-Nagumo model
}

\author{
Jun Zhang ${ }^{1 \dagger}$, Shimin Lin ${ }^{2 \dagger}$, Zixin Liu ${ }^{3 \dagger}$ and Fubiao Lin $^{3 *}$
}

\section{"Correspondence:}

linfubiao0851@163.com

${ }^{3}$ School of Mathematics and

Statistical, Guizhou University of

Finance and Econmics, Guiyang, China

Full list of author information is available at the end of the article

†Equal contributors

\begin{abstract}
In this work, we study the numerical approximation for the space fractional FitzHugh-Nagumo model. The numerical scheme is based on the Crank-Nicolson $(\mathrm{C}-\mathrm{N})$ method in time and Legendre-spectral method in space. In addition, we prove that the numerical scheme is unconditionally stable. Numerical examples are presented to verify validity of the proposed scheme.
\end{abstract}

MSC: $65 \mathrm{~N} 15 ; 65 \mathrm{~N} 30$

Keywords: FitzHugh-Nagumo model; Space fractional; Unconditionally stable; Legendre-spectral method

\section{Introduction}

As a important model in describing a prototype of an excitable system, the FitzHughNagumo model [1] has received great attention in recent years. The space fractional FitzHugh-Nagumo model is obtained by replacing standard Laplacian operator in FitzHugh-Nagumo model by Riesz derivatives. The purpose of introducing the spatial fractional FitzHugh-Nagumo model is mainly due to the fact that a spatial fractional derivative can capture the spatial connectivity of the extracellular domain more accurately.

Bu et al. [2] presented an ADI finite-element method to solve FitzHugh-Nagumo model. In [3], Liu et al. constructed a shifted Grünwald-Letnikov scheme to discretize the Riesz derivative of the fractional FitzHugh-Nagumo model. However, both the finite-element and finite-difference methods will produce large dense matrices due to the nonlocal term discretization when solving the linear systems. Yang et al. [4] and Cattani [5] proposed a fractional derivative of sinc function without singular kernel. In recent work [6-9], some general fractional calculus operators involving constant and variable order derivatives were used. Kumar et al. [10,11] and Singh [12] studied the fractional exothermic reaction model with Mittag-Leffler law. The fractional Laplace decomposition technique was used to investigate the numerical solution of that model. More results on numerical solutions of fractional derivatives can be found in [13-15].

This paper uses the Legendre-spectral method to handle nonlocal terms. We propose an efficient numerical scheme to solve the spatial fractional FitzHugh-Nagumo model, the numerical scheme is performed by combining it with a second order method in time

(c) The Author(s) 2019. This article is distributed under the terms of the Creative Commons Attribution 4.0 International License (http://creativecommons.org/licenses/by/4.0/), which permits unrestricted use, distribution, and reproduction in any medium, provided you give appropriate credit to the original author(s) and the source, provide a link to the Creative Commons license, and indicate if changes were made. 
and Legendre-spectral method in space. Moveover, we prove that the obtained numerical scheme is unconditionally stable.

In Sect. 2, we will introduce the spatial fractional FitzHugh-Nagumo model. In Sect. 3, the numerical scheme and stability analysis are studied. In Sect. 4, numerical experiments are performed to demonstrate the effectiveness of the numerical methods. The conclusion of this article is given in last section.

\section{FitzHugh-Nagumo model}

We consider the following fractional FitzHugh-Nagumo model:

$$
\begin{cases}u_{t}=\kappa \Delta^{\alpha / 2} u+u(1-u)(u-a)-v & \text { in } \Omega_{d} \times[0, T], \\ v_{t}=\beta u-\gamma v+\eta & \text { in } \Omega_{d} \times[0, T], \\ u(\cdot, 0)=u_{0}, v(\cdot, 0)=v_{0} & \text { in } \Omega_{d} \times[0, T], \\ u=0 & \text { on } \partial \Omega_{d} \times[0, T],\end{cases}
$$

where $1.5 \leq \alpha \leq 2, \Omega_{d}=(-1,1)^{d}, d=2,3$, and we define the following space fractional Laplace operator (see [16]):

$$
-\Delta^{\alpha}:=-\frac{1}{4} \sum_{j=1}^{d}\left(D_{x_{j}}^{\alpha}-{ }_{x_{j}} D^{\alpha}\right)\left({ }^{C} D_{x_{j}}^{\alpha}-{ }_{x_{j}}^{C} D^{\alpha}\right),
$$

where $D_{x_{j}}^{\alpha}, x_{j} D_{1}^{\alpha},{ }^{C} D_{x_{j}}^{\alpha},{ }_{x_{j}}{ }^{C} D_{1}^{\alpha}$ are left and right Riemann-Liouville and Captuo fractional derivatives, respectively. It is worth mentioning that our derivative is an extension of the Caputo and Riemann-Liouville derivative, so it contains a singular kernel around 0. But Yang-Srivastava-Machado fractional derivative does not contain a singular kernel.

We use $A \lesssim B$ to mean that $A \leq c B$, and $A \simeq B$ to mean that $A \lesssim B$ and $B \lesssim A$.

Lemma 1 ([17]) For $0<s<1, s \neq \frac{1}{2}$, if $w, v \in H_{0}^{s}(\Lambda)$, then

$$
\begin{array}{ll}
\left({ }_{1} D_{x-1}^{s} D_{x}^{s} u, v\right)_{\Lambda}=\left({ }_{-1} D_{x}^{s} u,{ }_{x} D_{1}^{s} v\right)_{\Lambda}, & \left({ }_{x} D_{1 x}^{s} D_{1}^{s} u, v\right)_{\Lambda}=\left({ }_{x} D_{1}^{s} u,{ }_{-1} D_{x}^{s} v\right)_{\Lambda}, \\
\left({ }_{x} D_{1-1}^{s} D_{x}^{s} u, v\right)_{\Lambda}=\left({ }_{-1} D_{x}^{s} u,{ }_{-1} D_{x}^{s} v\right)_{\Lambda}, & \left({ }_{-1} D_{x x}^{s} D_{1}^{s} u, v\right)_{\Lambda}=\left({ }_{x} D_{1}^{s} u,{ }_{x} D_{1}^{s} v\right)_{\Lambda} .
\end{array}
$$

Lemma 2 ([17]) Given $s>0, s \neq n-\frac{1}{2}, n \in \mathbb{N}$, for $w, v \in H_{0}^{s}(\Lambda)$, we have

$$
\left({ }_{-1} D_{x-1}^{s} D_{x}^{s} u, v\right)_{\Lambda} \cong \cos (\pi s)\left\|_{-1} D_{x}^{s} v\right\|_{L^{2}(\Lambda)}^{2} \cong \cos (\pi s)\left\|_{x} D_{1}^{s} v\right\|_{L^{2}(\Lambda)}^{2} \cong \cos (\pi s)\|v\|_{s, \Lambda}^{2} .
$$

From Lemmas 1 and 2, we obtain a bilinear form as follows:

$$
\begin{aligned}
a(u, w):= & -\frac{1}{4} \sum_{j=1}^{d}\left[\left({ }_{-1} D_{x_{j}}^{\alpha} u, x_{j} D_{1}^{\beta} w\right)+\left({ }_{x_{j}} D_{1}^{\alpha} u,{ }_{-1} D_{x_{j}}^{\alpha} w\right)\right. \\
& \left.-\left({ }_{-1} D_{x_{j}}^{\alpha} u,{ }_{-1} D_{x_{j}}^{\alpha} w\right)-\left({ }_{x_{j}} D_{1}^{\alpha} u,{ }_{x_{j}} D_{1}^{\alpha} w\right)\right] .
\end{aligned}
$$

It is easy to check that $\|u\|_{H^{\alpha}}^{2} \lesssim a(u, u)$. 
Theorem 1 Suppose that $(u, v) \in H_{0}^{\alpha}(\Omega)$, then we have the following estimates:

For $\gamma \geq 1$,

$$
\|u\|^{2}+\frac{1}{\beta}\|v\|^{2} \leq e^{-t}\left(\left\|u_{0}\right\|^{2}+\frac{1}{\beta}\left\|v_{0}\right\|^{2}\right)+\left(\frac{\eta^{2}}{\gamma \beta}+\left(\frac{a^{2}+2}{2}\right)^{2}\right)|\Omega| .
$$

For $1>\gamma>0$,

$$
\|u\|^{2}+\frac{1}{\beta}\|v\|^{2} \leq\left\|u_{0}\right\|^{2}+\frac{1}{\beta}\left\|v_{0}\right\|^{2}+\left(\frac{\eta^{2}}{\gamma \beta}+\left(\frac{a^{2}+2}{2}\right)^{2}\right)|\Omega| t .
$$

Proof Taking the inner product of the first equation of (2) with $u(t)$ and of second equation with $\frac{1}{\beta} v(t)$, we have

$$
\frac{1}{2}\left(\frac{d}{d t}\|u\|^{2}+\frac{1}{\beta} \frac{d}{d t}\|v\|^{2}+2 \kappa\left\|D^{\frac{\alpha}{2}} u\right\|^{2}\right)=(u(1-u)(u-a), u)+(-\gamma v+\eta, v) .
$$

Note that

$$
\begin{aligned}
-u^{4}+(a+1) u^{3}-a u^{2} & =-\frac{1}{2} u^{2}\left[(u-a-1)^{2}+u^{2}-\left(a^{2}+1\right)\right], \\
& =-\frac{1}{2} u^{2}\left[(u-a-1)^{2}\right]-\frac{1}{2}\left[\left(u^{2}-\frac{a^{2}+2}{2}\right)^{2}+u^{2}-\left(\frac{a^{2}+2}{2}\right)^{2}\right], \\
-\gamma v^{2}+\eta v & =-\frac{\gamma}{2}\left(v^{2}+\frac{2 \eta}{\gamma} v+\left(\frac{\eta}{\gamma}\right)^{2}+v^{2}-\left(\frac{\eta}{\gamma}\right)^{2}\right) \\
= & -\frac{\gamma}{2}\left(v+\frac{\eta}{\gamma}\right)^{2}-\frac{\gamma}{2} v^{2}+\frac{\eta^{2}}{2 \gamma} .
\end{aligned}
$$

Then, we have

$$
\frac{d}{d t}\|u\|^{2}+\frac{1}{\beta} \frac{d}{d t}\|v\|^{2}+\|u\|^{2}+\frac{\gamma}{\beta}\|v\|^{2} \leq\left(\frac{\eta^{2}}{\gamma \beta}+\left(\frac{a^{2}+2}{2}\right)^{2}\right)|\Omega| .
$$

This completes the proof.

\section{Numerical scheme and stability analysis}

In this part, we will propose a $\mathrm{C}-\mathrm{N}$ scheme in time, and we will also discuss the unconditional stability of our numerical scheme. First, we define the time step $\delta t=T / M$, where $M$ is a positive integer, $t_{n}=n \delta t, 0 \leq n \leq M-1$. Consider the second-order time-discrete scheme as follows:

$$
\left\{\begin{array}{l}
\frac{u^{n+1}-u^{n}}{\delta t}=-\kappa(-\Delta)^{\alpha / 2} u^{n+\frac{1}{2}}+u^{n+\frac{1}{2}}\left(1-u^{*}\right)\left(u^{*}-a\right)-v^{n+\frac{1}{2}}, \\
\frac{v^{n+1}-v^{n}}{\delta t}=\beta u^{n+\frac{1}{2}}-\gamma v^{n+\frac{1}{2}}+\eta
\end{array}\right.
$$

where $u^{*}=\frac{3}{2} u^{n}-\frac{1}{2} u^{n-1}$, for $n \geq 1$, and $u^{*}=u^{0}$, for $n=0$.

Theorem 2 The time discrete scheme (5) is unconditionally stable, and we have

$$
\left\|u^{k+1}\right\|^{2}+\frac{1}{\beta}\left\|v^{k+1}\right\|^{2} \lesssim\left\|u^{0}\right\|^{2}+\left\|v^{0}\right\|^{2}+1
$$


Proof Taking the inner product of (5) with $\delta t\left(u^{n+1}+u^{n}\right)$ and $\frac{1}{\beta} \delta t\left(v^{n+1}+v^{n}\right)$, respectively, we get

$$
\begin{gathered}
\left\|u^{n+1}\right\|^{2}-\left\|u^{n}\right\|^{2}+\frac{1}{\beta}\left(\left\|v^{n+1}\right\|^{2}-\left\|v^{n}\right\|^{2} \|\right)+2 \kappa\left\|D^{\frac{\alpha}{2}} u^{n+\frac{1}{2}}\right\|^{2} \\
=-\delta t\left(\left\|u^{n+\frac{1}{2}}\left(u^{*}-a-1\right)\right\|^{2}+\left\|u^{n+\frac{1}{2}} u^{*}\right\|^{2}-\left(a^{2}+1\right)\left\|u^{n+\frac{1}{2}}\right\|^{2}\right) \\
-\delta t\left(\frac{\gamma}{\beta}\left\|v^{n+\frac{1}{2}}+\frac{\eta}{\gamma}\right\|^{2}-\frac{\gamma}{\beta}\left\|v^{n+\frac{1}{2}}\right\|^{2}+\frac{\eta^{2}}{\beta \gamma}|\Omega|\right) .
\end{gathered}
$$

Dropping some positive terms and summing up over $n=1,2, \ldots, k$, we get

$$
\left\|u^{k+1}\right\|^{2}+\frac{1}{\beta}\left\|v^{k+1}\right\|^{2} \leq \delta t\left(a^{2}+1\right) \sum_{n=1}^{k}\left\|u^{n+\frac{1}{2}}\right\|^{2}+\left(\left\|u^{1}\right\|+\frac{1}{\beta}\left\|v^{1}\right\|^{2}+\frac{\eta^{2} T}{\beta \gamma}|\Omega|\right) .
$$

Using discrete Gronwall lemma, one has

$$
\left\|u^{k+1}\right\|^{2}+\frac{1}{\beta}\left\|v^{k+1}\right\|^{2} \lesssim\left\|u^{1}\right\|+\frac{1}{\beta}\left\|v^{1}\right\|^{2}+1
$$

Linking the first step, we can prove that

$$
\left\|u^{1}\right\|^{2}+\frac{1}{\beta}\left\|v^{1}\right\|^{2} \lesssim\left\|u^{0}\right\|^{2}+\left\|v^{0}\right\|^{1}+1
$$

This ends the proof.

\section{Numerical implementation and numerical results}

We consider the Legendre-spectral method to discretize in the spatial direction. We obtain the full discrete scheme of problem (5) as follows:

$$
\left\{\begin{array}{l}
\left(\frac{u_{N}^{n+1}-u_{N}^{n}}{\delta t}, \phi_{N}\right)=-\kappa a\left(u_{N}^{n+\frac{1}{2}}, \phi_{N}\right)+\left(u_{N}^{n+\frac{1}{2}}\left(1-u_{N}^{*}\right)\left(u_{N}^{*}-a\right)-v_{N}^{n+\frac{1}{2}}, \phi_{N}\right), \quad \phi_{N} \in \mathbb{P}_{N} \\
\left(\frac{v_{N}^{n+1}-v_{n}^{n}}{\delta t}, \varphi_{N}\right)=\beta\left(u_{N}^{n+\frac{1}{2}}, \varphi_{N}\right)-\gamma\left(v_{N}^{n+\frac{1}{2}}, \varphi_{N}\right)+\left(\eta, \varphi_{N}\right), \quad \varphi_{N} \in \mathbb{P}_{N} .
\end{array}\right.
$$

Define

$$
u_{N}^{n}=\sum_{i, j=1}^{N-1} u_{i, j}^{n} L_{N, i}(x) L_{N, j}(y), v_{N}^{n}=\sum_{i, j=1}^{N-1} v_{i, j}^{n} L_{N, i}(x) L_{N, j}(y)
$$

where $L_{N, i}(x)$ are Lagrangian polynomials, $u_{i, j}^{n}$ denote the unknown coefficients.

In order to verify the asymptotic behavior of the solutions, the effect of space-fractional derivative $\alpha$ will also be investigated. The numerical method (7) is computed in the square $[0,2.5] \times[0,2.5]$ with $\kappa=10^{-4}, \mu=0.1, \beta=5 \times 10^{-3}, \gamma=10^{-2}$, and $\eta=0$. Tables $1-4$ display the temporal convergence orders and the errors in the $L^{2}$ and $H^{\alpha / 2}$ norms for $\alpha=1.5$ and 1.7. It is confirmed that our numerical scheme can achieve second-order accuracy in time, and the numerical solutions are in good agreement with the exact solution. In addition, using scheme (7), we simulate the dynamic behavior for different $\alpha$, where the results are summarized in Fig. 1. As can be seen from this figure, due to the long-tail 
Table 1 The $L^{2}$ and $H^{\alpha / 2}$ numerical errors at $\alpha=1.5$ for various temporal resolutions $u$

\begin{tabular}{llll}
\hline$\Delta t$ & $L^{2}$-error & $H^{\alpha / 2}$-error & Order \\
\hline 0.1 & 0.002853104 & 0.005747130 & - \\
0.05 & 0.000741835 & 0.001483160 & 1.98009 \\
0.01 & $3.06393 \mathrm{E}-05$ & $6.08969 \mathrm{E}-05$ & 1.99413 \\
0.005 & $7.53784 \mathrm{E}-08$ & $1.52751 \mathrm{E}-05$ & 2.0059 \\
0.001 & $2.96203 \mathrm{E}-09$ & $6.04827 \mathrm{E}-07$ & 2.01242 \\
0.0005 & $6.98812 \mathrm{E}-10$ & $1.51197 \mathrm{E}-07$ & 2.00017 \\
\hline
\end{tabular}

Table 2 The $L^{2}$ and $H^{\alpha / 2}$ numerical errors at $\alpha=1.5$ for various temporal resolutions $V$

\begin{tabular}{llll}
\hline$\Delta t$ & $L^{2}$-error & $H^{\alpha / 2}$-error & Order \\
\hline 0.1 & $1.27887 \mathrm{E}-05$ & $2.51306 \mathrm{E}-05$ & - \\
0.05 & $3.41154 \mathrm{E}-06$ & $6.67129 \mathrm{E}-06$ & 1.90636 \\
0.01 & $1.43796 \mathrm{E}-07$ & $2.80067 \mathrm{E}-07$ & 1.96747 \\
0.005 & $3.62094 \mathrm{E}-08$ & $7.04652 \mathrm{E}-08$ & 1.98958 \\
0.001 & $1.46667 \mathrm{E}-09$ & $2.84137 \mathrm{E}-09$ & 1.99219 \\
0.0005 & $3.90331 \mathrm{E}-10$ & $7.32173 \mathrm{E}-10$ & 1.90986 \\
\hline
\end{tabular}

Table 3 The $L^{2}$ and $H^{\alpha / 2}$ numerical errors at $\alpha=1.7$ for various temporal resolutions $u$

\begin{tabular}{llll}
\hline$\Delta t$ & $L^{2}$-error & $H^{\alpha / 2}$-error & Order \\
\hline 0.1 & 0.00285274 & 0.006814937 & - \\
0.05 & 0.000741741 & 0.001731916 & 1.94336 \\
0.01 & $3.06355 \mathrm{E}-05$ & $7.10406 \mathrm{E}-05$ & 1.98009 \\
0.005 & $7.69009 \mathrm{E}-06$ & $1.78184 \mathrm{E}-05$ & 1.99413 \\
0.001 & $3.01524 \mathrm{E}-07$ & $7.06222 \mathrm{E}-07$ & 2.01241 \\
0.0005 & $7.53763 \mathrm{E}-08$ & $1.76539 \mathrm{E}-07$ & 2.00008 \\
\hline
\end{tabular}

Table 4 The $L^{2}$ and $H^{\alpha / 2}$ numerical errors at $\alpha=1.7$ for various temporal resolutions $v$

\begin{tabular}{llll}
\hline$\Delta t$ & $L^{2}$-error & $H^{\alpha / 2}$-error & Order \\
\hline 0.1 & $1.2788 \mathrm{E}-05$ & $2.92707 \mathrm{E}-05$ & - \\
0.05 & $3.41136 \mathrm{E}-06$ & $7.76832 \mathrm{E}-06$ & 1.90635 \\
0.01 & $1.43788 \mathrm{E}-07$ & $3.25984 \mathrm{E}-07$ & 1.96741 \\
0.005 & $3.62076 \mathrm{E}-08$ & $8.20116 \mathrm{E}-08$ & 1.98958 \\
0.001 & $1.46662 \mathrm{E}-09$ & $3.30567 \mathrm{E}-09$ & 1.99224 \\
0.0005 & $3.90343 \mathrm{E}-10$ & $8.49135 \mathrm{E}-10$ & 1.90973 \\
\hline
\end{tabular}
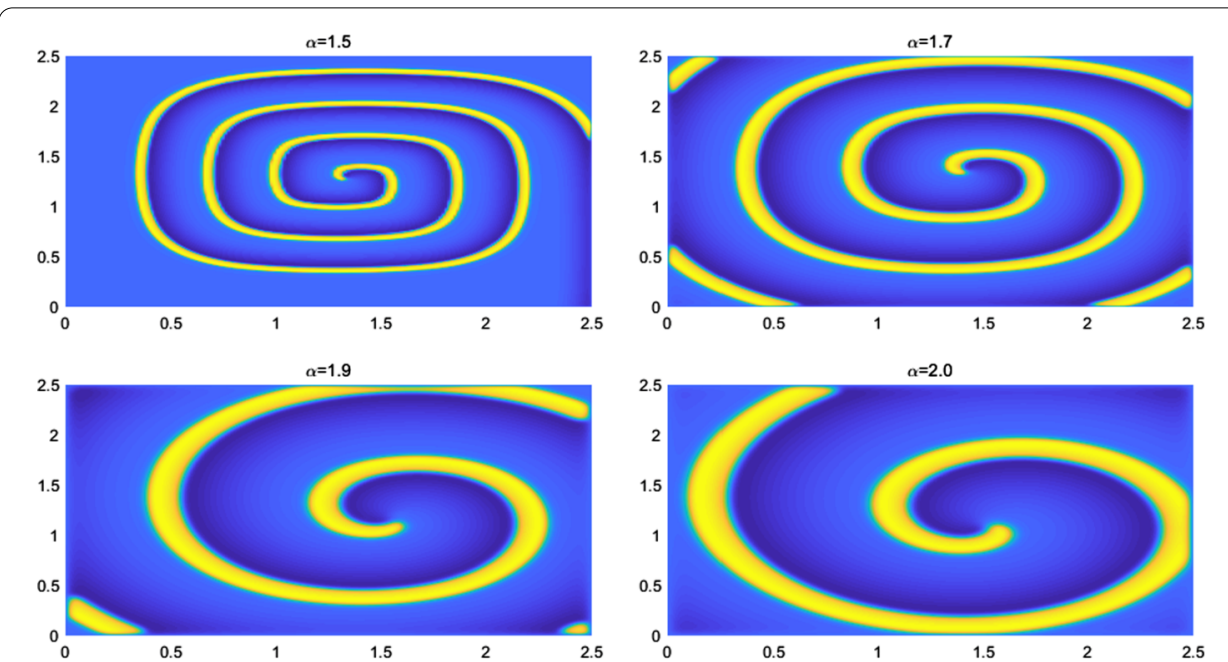

Figure 1 The dynamic behavior for different $\alpha$ 
mechanism of the fractional Laplace operator, the wavelength becomes larger when the fractional diffusion coefficient $\alpha$ becomes larger. This shows that a fractional equation with diffusion mechanisms is a powerful tool to describe dynamic state.

\section{Conclusions}

An efficient linearized numerical scheme is constructed to solve the space fractional FitzHugh-Nagumo equation. The numerical scheme is proved to be stable. Numerical examples show that the proposed scheme is effective. Moreover, the fractional diffusion coefficient $\alpha$ has a significant effect on the dynamic behavior.

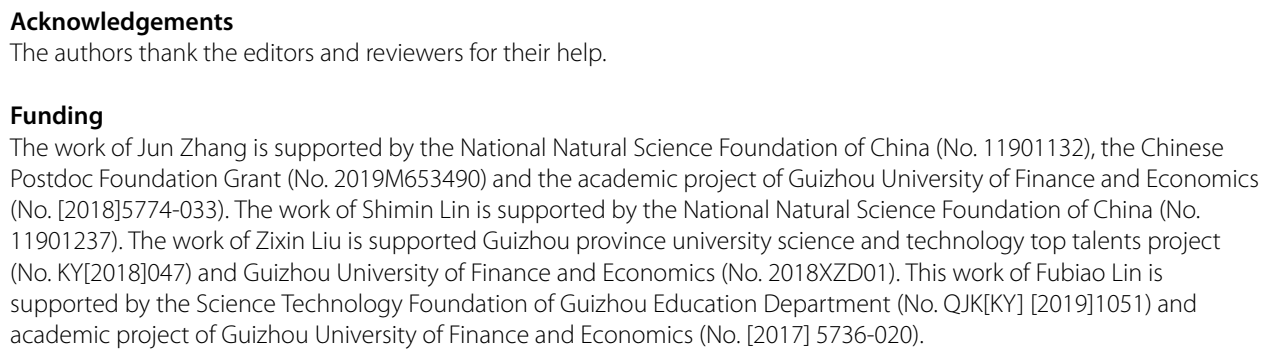

\section{Authors' information}

Jun Zhang, Computational mathematics research center, Guizhou University of Finance and Economics, Guiyang 550025, China. E-mail addresses: jzhang@mail.gufe.edu.cn. Shimin Lin, Department of Science, Jimei University, Xiamen, Fujian 361021, China. E-mail addresses: smlin@jmu.edu.cn. Zixin Liu, School of Mathematical Sciences, Guizhou University of Finance and Economics, Guiyang 550025, China. E-mail addresses: xinxin905@163.com. Fubiao Lin, Corresponding author, School of Mathematical Sciences, Guizhou University of Finance and Economics, Guiyang 550025, China. E-mail addresses: linfubiao0851@163.com.

\section{Author details}

'Computational Mathematics Research Center, Guizhou University of Finance and Economics, Guiyang, China. ${ }^{2}$ Department of Science, Jimei University, Fujian, China. ${ }^{3}$ School of Mathematics and Statistical, Guizhou University of Finance and Econmics, Guiyang, China.

\section{Publisher's Note}

Springer Nature remains neutral with regard to jurisdictional claims in published maps and institutional affiliations.

Received: 3 April 2019 Accepted: 28 July 2019 Published online: 20 August 2019

References

1. Fitzhugh, R.: Impulses and physiological states in theoretical models of nerve membrane. Biophys. J. 1(6), 445-466 (1961)

2. Bu, W., Tang, Y., Wu, Y., Yang, J.: Crank-Nicolson ADI Galerkin finite element method for two-dimensional fractional FitzHugh-Nagumo monodomain model. Appl. Math. Comput. 257, 355-364 (2015)

3. Liu, F., Turner, I., Anh, V., Yang, Q., Burrage, K.: A numerical method for the fractional FitzHugh-Nagumo monodomain model. ANZIAM J. 54, C608-C629 (2012) 
4. Yang, X.J., Gao, F., Machado, J.A.T., Baleanu, D.: A new fractional derivative involving the normalized sinc function without singular kernel. Eur. Phys. J. Spec. Top. 226(16-18), 3567-3575 (2017)

5. Cattani, C.: Sinc-fractional operator on Shannon wavelet space. Front. Phys. 6, 118 (2018)

6. Yang, X.J., Gao, F., Ju, Y., Zhou, H.W.: Fundamental solutions of the general fractional-order diffusion equations. Math. Methods Appl. Sci. 41(18), 9312-9320 (2018)

7. Yang, X.J.: General Fractional Derivatives: Theory, Methods and Applications. CRC Press, New York (2019)

8. Gao, F:: General fractional calculus in nonsingular power-law kernel applied to model anomalous diffusion phenomena in heat-transfer problems. Therm. Sci. 21, S11-S18 (2017)

9. Yang, X.J.: Fractional derivatives of constant and variable orders applied to anomalous relaxation models in heat-transfer problems. Therm. Sci. 21(3), 1161-1171 (2017)

10. Kumar, D., Singh, J., Tanwar, K., Baleanu, D.: A new fractional exothermic reactions model having constant heat source in porous media with power, exponential and Mittag-Leffler laws. Int. J. Heat Mass Transf. 138, 1222-1227 (2019)

11. Singh, J., Kumar, D., Baleanu, D.: New aspects of fractional biswasmilovic model with Mittag-Leffler law. Fractional order mathematical models in physical sciences. Math. Model. Nat. Phenom. 14, 303 (2019)

12. Singh, J.: A new analysis for fractional rumor spreading dynamical model in a social network with Mittag-Leffler law. Chaos, Interdiscip. J. Nonlinear Sci. 29, 013137 (2019)

13. Singh, J., Kumar, D., Hammouch, Z., Atangana, A.: A fractional epidemiological model for computer viruses pertaining to a new fractional derivative. Appl. Math. Comput. 316, 504-515 (2017)

14. Goswami, A., Singh, J., Kumar, D., Sushila: An efficient analytical approach for fractional equal width equations describing hydro-magnetic waves in cold plasma. Phys. A, Stat. Mech. Appl. 524(15), 563-575 (2019)

15. Singh, J., Kumar, D., Baleanu, D., Rathore, S.: On the local fractional wave equation in fractal strings. Math. Methods Appl. Sci. 42(5), 1588-1595 (2019)

16. Lin, S., Azaïez, M., Xu, C.: A fractional Stokes equation and its spectral approximation. Int. J. Numer. Anal. Model. 15(1), $170-192(2018)$

17. Li, X., Xu, C.: A space-time spectral method for the time fractional diffusion equation. SIAM J. Numer. Anal. 47(3), $2108-2131(2009)$

\section{Submit your manuscript to a SpringerOpen ${ }^{\circ}$ journal and benefit from:}

- Convenient online submission

- Rigorous peer review

- Open access: articles freely available online

- High visibility within the field

Retaining the copyright to your article

Submit your next manuscript at $\boldsymbol{\nabla}$ springeropen.com 